\title{
A co-ordinate system independent streamwise upwind method for fluid flow computation
}

\author{
Joel H. Ferziger and Yu-heng Tseng*, $\dagger$ \\ Flow Physics \& Computation, Mechanical Engineering Department, Stanford University, Building 500, \\ Stanford, CA 94305-3030, U.S.A.
}

\begin{abstract}
SUMMARY
A new co-ordinate invariant streamwise upwind formulation for convection dominated flows is developed. The eddy diffusivity/viscosity is added directly to the equations in order to remove the oscillations in the solution. The equations then can be solved by any high-order scheme and the solution retains the accuracy of the high-order scheme. The accuracy and reduced lateral thickness growth rate are demonstrated with several numerical examples, including pure convective flows and lid-driven cavity flow. The lateral spreading due to the numerical diffusion is controlled by the anisotropic tensor. Copyright (C) 2004 John Wiley \& Sons, Ltd.
\end{abstract}

KEY WORDS: anisotropic diffusivity; co-ordinate invariant; SUPG

\section{INTRODUCTION}

It is well known that although central difference approximations are more accurate than upwind biased approximations that use the same number of grid points, they are prone to produce solutions that contain unphysical (and mathematically incorrect) oscillations. Furthermore, when they are used in conjunction with certain iterative or time advance methods, they are less stable than upwind methods. The generally accepted criterion for the onset of oscillations ('wiggles') in the solutions is that the cell Reynolds (or Peclet) number should be greater than two. It should be emphasized that this is a necessary but not a sufficient condition.

A commonly used means of avoiding or, at least reducing, the severity of oscillations in solutions is to use upwind biased methods. Indeed, one can show [1] that the only method guaranteed to produce monotone solutions is the first-order accurate upwind method. However, upwind methods produce smooth solutions by introducing significant diffusive errors. They also tend to be more stable when used with iterative methods; the additional stability can be traced to the same source.

*Correspondence to: Yu-heng Tseng, Department of Mechanical Engineering, The Johns Hopkins University, 223 Latrobe Hall, Baltimore, MD 21218, U.S.A.

†E-mail: yhtseng@pegasus.me.jhu.edu 


\section{J. H. FERZIGER AND Y.-H. TSENG}

When upwind methods are used in two or three dimensions, the diffusive error usually produces an unphysical increase in the rate of growth of thin layers of rapid lateral variation of the solution e.g. thin shear layers such as boundary layers or jets or thin layers of material due to localized sources. In oceanic simulations, this effect may lead to broadening and reduction of strength of coastal currents [2]. In atmospheric work, it may result in broadening of upper atmosphere jets. Thus, although upwind methods produce smooth results, they modify the solution substantially, often in ways that degrade the quality significantly.

One means of overcoming this problem is to use a method that introduces diffusion only in the streamwise (or, as it is often called, streamline) direction. Such a method will produce unphysical effects in regions of strong streamwise variation (such as stagnation points) but can eliminate the undesirable lateral spreading mentioned above. Methods of this type are not new and several have been proposed in the past. We now review some of them.

Brooks and Hughes [3] proposed a streamline upwind Petrov-Galerkin (SUPG) method that achieves the desired effect by introducing an upwind biased weighting in a variationally based finite element method. This method has been adopted by a number of authors. Kelly et al. [4] introduced the anisotropic eddy viscosity using an balancing dissipation method. Raithby and his collaborators introduced a skew upwind method [5]. This method uses finite difference approximations along local streamline directions. However, to obtain the values needed in the difference approximation, interpolation is required and the diffusive interpolation error gives solutions that contain the kinds of effects that were mentioned above.

More recently, Large et al. [6] proposed an anisotropic eddy viscosity method specifically for the purpose of improving the prediction of equatorial currents in the ocean. Although their method is quite successful in accomplishing its desired aim (as they demonstrated), it is tied to the particular co-ordinate system they used and thus does not have the invariance properties or generality that a good method should possess.

In this paper, we offer an alternative approach in which a co-ordinate invariant anisotropic eddy viscosity is added to the equations of motion directly. The equations may then be solved by any method one desires, including central difference methods. The essential difference between the proposed approach and previous SUPG methods is the form of the anisotropy eddy viscosity, thus the method can be seen as a co-ordinate invariant SUPG method [3,4]. The accuracy mainly depends on the based numerical schemes (e.g. central difference). Because it is simpler, we shall discuss the scalar case first and then go on to the vector case. After introducing the method, we shall offer some examples that illustrate the effectiveness of the approach.

\section{AN ANISOTROPIC DIFFUSIVITY FOR A CONSERVED SCALAR}

The equation for the conservation of a conserved scalar, $\phi$, is well known

$$
\frac{\partial \phi}{\partial t}+u_{j} \frac{\partial \phi}{\partial x_{j}}=\frac{\partial}{\partial x_{j}}\left(\alpha \frac{\partial \phi}{\partial x_{j}}\right)
$$

All of the terms have been written in divergence (strong conservation) form to make the achievement of conservation in a numerical approximation easier. In particular, the term on the right-hand side is the negative of the divergence of the diffusive flux, $q_{j}=-\alpha \partial \phi / \partial x_{j}$. As noted in the introduction, when this equation is solved with an upwind method in two or 
three dimensions, the solution is smooth but the numerical errors produce greatly increased lateral spreading of a thin layer of the concentration, $\phi$.

In order to avoid the oscillations associated with central difference methods and the excessive diffusion of upwind methods, we would like to add to this equation a term that increases the diffusion in the streamwise direction without modifying the diffusion in the lateral directions. This is easily done. Such a flux has the form

$$
q_{j N}=-A_{N} u_{j} u_{k} \frac{\partial \phi}{\partial x_{k}}
$$

In vector notation, this is

$$
\mathbf{q}_{N}=-A_{N} \mathbf{u} \mathbf{u} \cdot \nabla \phi
$$

In essence, we have added a diffusive term in which the diffusivity is the tensor

$$
\alpha_{j k}=A_{N} u_{j} u_{k}=\alpha_{N} \frac{u_{j} u_{k}}{q^{2}}
$$

where $q^{2}=u_{m} u_{m}$ is the square of the magnitude of the local velocity. The added diffusive flux is designed such that, when these equations are transformed into a co-ordinate system in which one of the axes is aligned with the local velocity vector, only the streamwise component of the flux is non-zero i.e. the diffusivity tensor becomes in $2 \mathrm{D}$

$$
\alpha=\left(\begin{array}{ll}
\alpha & 0 \\
0 & 0
\end{array}\right)
$$

Thus, Equation (1) represents an additional diffusive contribution that is entirely in the streamwise direction. Note that the form of the anisotropic diffusivity tensor is very similar to that proposed in Reference [4]. However, the diffusion sources are totally different and are coordinate invariance.

We emphasize that the diffusive flux is added strictly for numerical reasons and has no physical significance. Indeed, it will, in some cases, cause the solution to be less accurate. To be sure that we do not introduce wiggles into the solution, the constant must be chosen such that the cell Peclet number obeys the condition

$$
P e_{\mathrm{c}}=\frac{q \Delta}{|\alpha|}<2
$$

This condition can easily be satisfied by choosing $A_{N}$ so that

$$
A_{N} \geqslant \frac{q \Delta}{2}
$$

In these inequalities, $\Delta$ should be chosen to be the largest distance within a cell i.e. the length of the diagonal $\left(\Delta x_{k} \Delta x_{k}\right)^{1 / 2}$. The best choice for the coefficient $A_{N}$ is obtained by replacing the inequality sign by an equality sign. In that way, the minimum amount of diffusion needed to achieve the desired result is introduced. This is a sufficient, but not necessary condition for boundedness of central difference schemes. If higher-order central difference scheme is used as the based scheme, the requirement for local Peclet number can be relaxed (e.g. $\left.P e_{\mathrm{c}}<4\right)$. 


\section{J. H. FERZIGER AND Y.-H. TSENG}

When the additional flux term is added to Equation (1), the resulting equation can be solved with any standard method. In particular, central difference methods should produce solutions with less lateral spreading and smaller oscillations when this term is added. If the solution is slowly varying in the streamwise direction, the effect of the added term should be nothing more than the reduction of oscillations and added stability to iterative numerical solution methods. Note that this approach does not guarantee monotonicity. We emphasize that no lateral diffusion has been added. Furthermore, the new term is quite easily evaluated. These claims will be validated in Section 4.

\section{THE NAVIER-STOKES EQUATIONS}

The same approach can be applied to the Navier-Stokes equations, the only substantial difference being that the conserved quantity is a vector and its flux is therefore a second rank tensor. This means that the added viscosity will need to be a fourth rank tensor.

The Navier-Stokes equations can be written as

$$
\frac{\partial u_{i}}{\partial t}+u_{j} \frac{\partial u_{i} u_{j}}{\partial x_{j}}=-\frac{1}{\rho} \frac{\partial p}{\partial x_{i}}+\frac{\partial}{\partial x_{j}} \tau_{i j}
$$

where

$$
\tau_{i j}=-v\left(\frac{\partial u_{i}}{\partial x_{j}}+\frac{\partial u_{j}}{\partial x_{i}}\right)=-2 v S_{i j}
$$

is the viscous momentum flux tensor and $S_{i j}$ is the rate of strain tensor.

In this case, a streamwise momentum flux takes the form

$$
\tau_{i j N}=-B_{N} u_{i} u_{j} u_{k} u_{l}\left(\frac{\partial u_{k}}{\partial x_{l}}+\frac{\partial u_{l}}{\partial x_{k}}\right)=-2 B_{N} u_{i} u_{j} u_{k} u_{l} S_{k l}
$$

When this is transformed into a co-ordinate system in which the local velocity vector is the 1 -axis, it is found that only $\tau_{11 N}$ is non-zero. The added viscosity is

$$
v_{i j k l}=B_{N} u_{i} u_{j} u_{k} u_{l}=C_{N} \frac{u_{i} u_{j} u_{k} u_{l}}{q^{4}}
$$

The condition that the coefficient $C_{N}$ must satisfy so as to assure the absence of wiggles is

$$
C_{N} \geqslant \frac{q \Delta}{2}
$$

in analogy with Equation (7).

As in the scalar case, the modified equation obtained by adding the streamwise viscous term to the Navier-Stokes equations can be solved with any numerical method and central difference methods are preferred. As in the scalar case, the coefficient $C_{N}$ should be chosen so that the inequality sign is replaced by an equality sign.

Although it appears a bit formidable, the new term is actually quite easily computed. The term $u_{k} u_{l} S_{k l}$ is simply the double scalar product of the rate of strain tensor with the velocity vector and is needed in all of the terms. It should therefore be computed first. In each equation, this quantity is then multiplied by $u_{i} u_{j}$ and added to the other viscous terms. 
CO-ORDINATE SYSTEM INDEPENDENT STREAMWISE UPWIND METHOD

In the following section, we shall apply the streamwise diffusion terms to the computation of some standard model problems.

\section{NUMERICAL EXAMPLES}

\subsection{Scalar transport in a constant convective flow}

A typical test for the convection discretization is to consider the problem of transport of a scalar quantity in a known velocity field. We discuss the behaviour of the anisotropic upwinding method using both steady and unsteady solutions. A step profile is convected in a uniform flow oblique to grid line. Since only convection is present in this case, the equation to be solved is

$$
\frac{\partial \phi}{\partial t}=u \frac{\partial \phi}{\partial x}+v \frac{\partial \phi}{\partial y}
$$

In the current test, the velocity is given by $u=v=u_{0}$ (const) which means that the scalar is transported along a $45^{\circ}$ diagonal. The domain size is $L \times W=\left[\begin{array}{ll}0 & 1\end{array}\right] \times\left[\begin{array}{ll}0 & 1\end{array}\right]$. The scalar $\phi$ at west boundary $(x=0)$ is prescribed as a step function

$$
\phi(y)= \begin{cases}0 & y<0.2 \\ 1 & y \geqslant 0.2\end{cases}
$$

and $\phi$ at south boundary $(y=0)$ is set to zero.

4.1.1. Steady case. The scalar distribution does not vary with time in a steady case $(\partial \phi / \partial t=0)$. At the outflow boundaries, the first-order upwind scheme is used in all cases and the simulation domain is extended further downstream so that the effect of outflow condition is not shown. We show results obtained using the upwind difference scheme (UDS), streamwise upwind difference scheme (SUDS) and central difference scheme (CDS) below. In Figure 1, we show profiles of the scalar $\phi$ at $y=0.5$, obtained on uniform $10 \times 10$ and $20 \times 20$ grids. The effect of severe numerical diffusion is clearly seen in the UDS solution. Only a little improvement is found in the solution on the refined grid. On the other hand, SUDS produces a profile that is steeper, but it has a mild overshoot. CDS generates severe oscillations. The numerical diffusion introduced by these schemes can be seen in the contour plots of the scalar $\phi$, see Figures 2 and 3. No numerical diffusion is found in the CDS results but the introduced oscillations are clearly convected in the streamwise direction.

Note that the streamwise upwind method does not produce zero lateral spreading of the layer. The reason for this is that the numerical errors introduced when the streamwise diffusion term is evaluated with second-order central differences has the form of a fourth-order diffusion term. Such a term should be expected to produce a lateral width that is proportional to $s^{1 / 4}$ where $s$ is the downstream distance (Figure 4). This compares with the $s^{1 / 2}$ behaviour of second-order diffusion (the dash line in Figure 4). The layer thickness $2 \delta$ is then proportional to $\left(s_{0}+s\right)^{1 / 4}$, where $s_{0}$ is the initial layer thickness. In Figure 4, layer width $\delta$ for UDS $(\diamond)$ and SUDS (०) results is expressed in terms of the distance between the $\phi=0.1$ and $\phi=0.9$ contours. The solid lines correspond to the fitting function $\delta=c\left(s_{0}+s\right)^{1 / 4}$, where 


\section{J. H. FERZIGER AND Y.-H. TSENG}
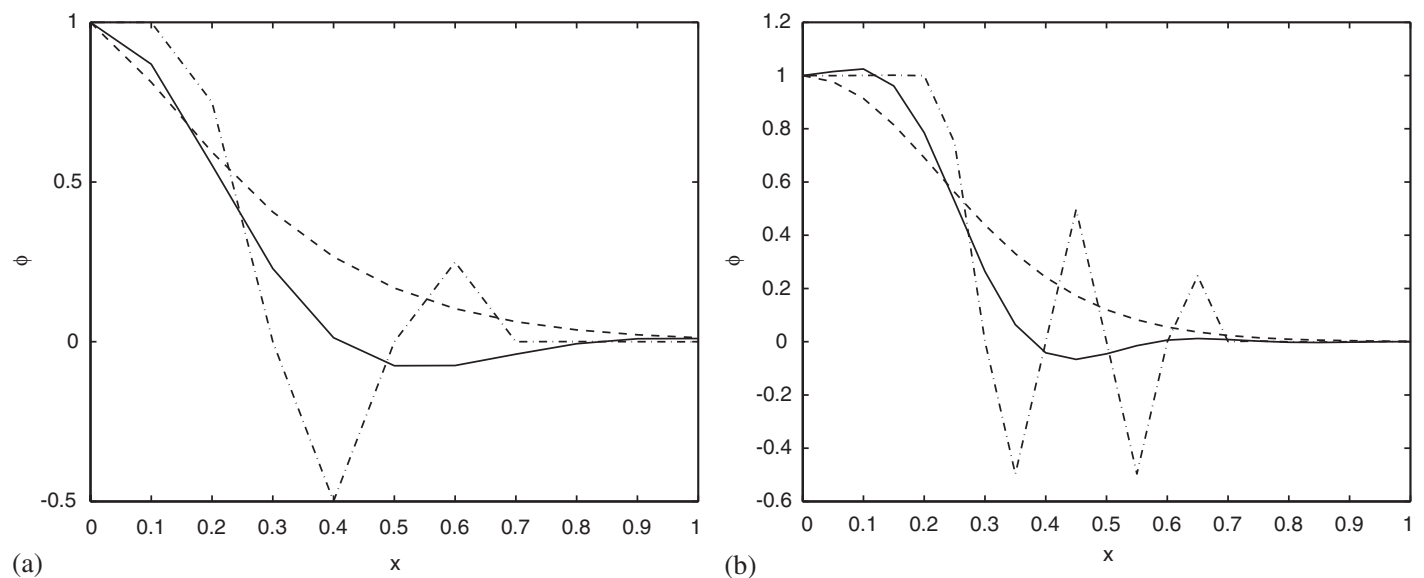

Figure 1. Profile of scalar $\phi$ at $y=5$ (--: UDS, - : CDS and $-:$ SUDS), calculated on a $10 \times 10$ grid (a) and a $20 \times 20$ grid (b). The exact solution is a step function where the step is located at $y=0.3$.

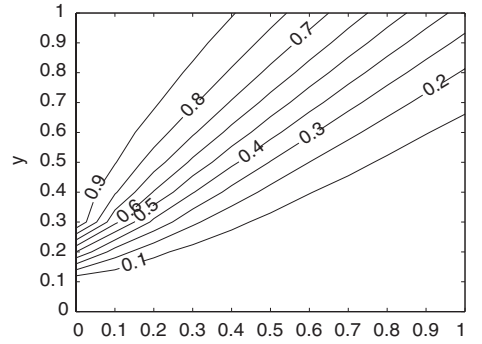

(a)

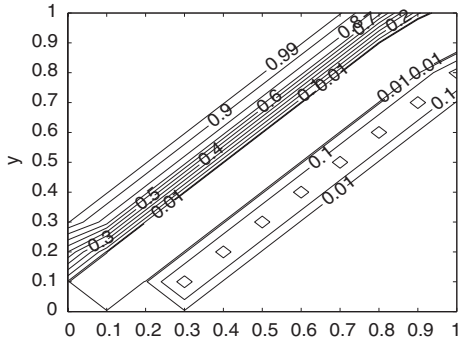

(b)

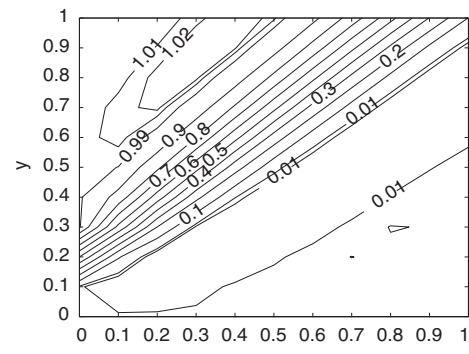

(c)

Figure 2. Contour plot of the scalar $\phi$ for a constant convective flow on a $10 \times 10$ grid: (a) UDS; (b) CDS; and (c) SUDS.

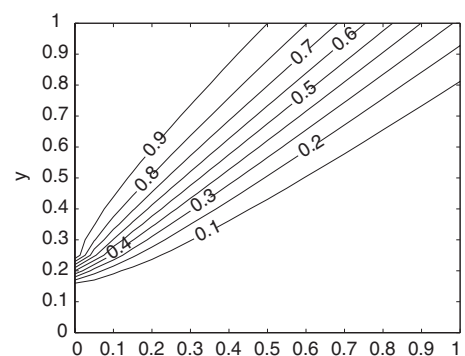

(a)

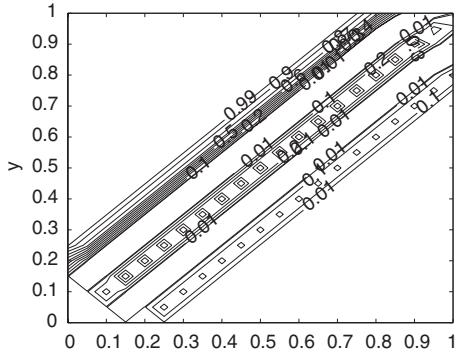

(b)

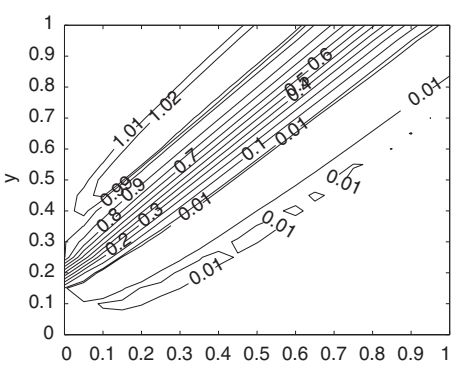

(c)

Figure 3. Contour plot of the scalar $\phi$ for a constant convective flow on a $20 \times 20$ grid: (a) UDS; (b) CDS; and (c) SUDS. 

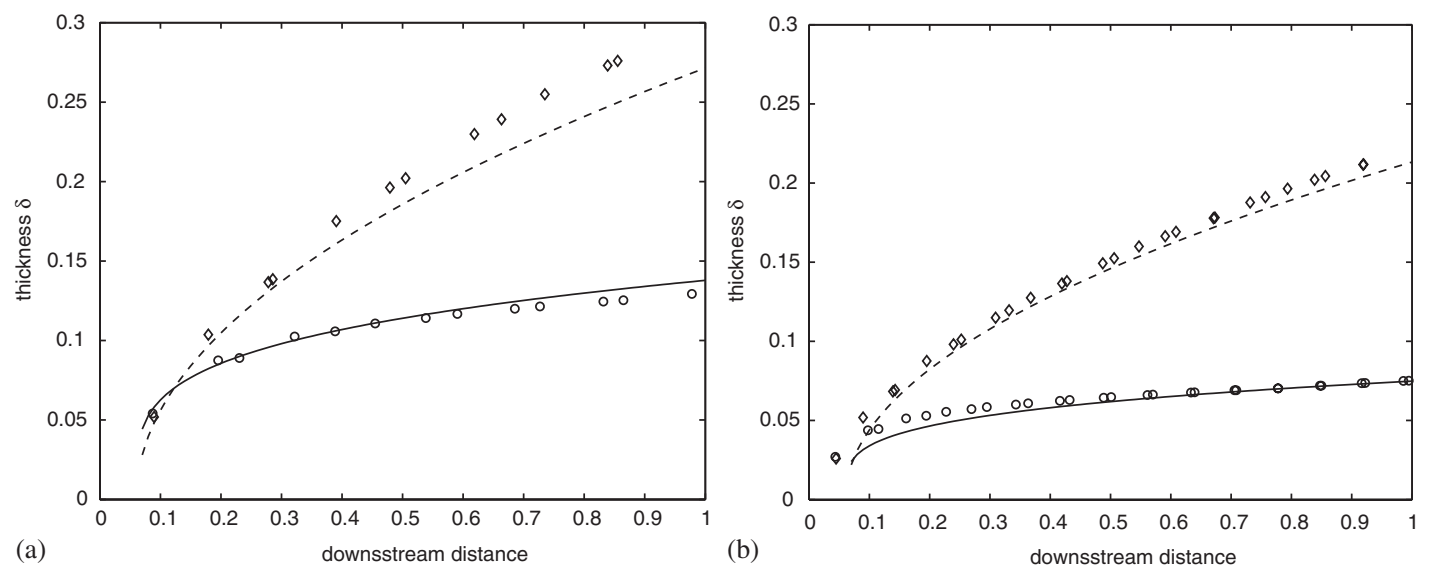

Figure 4. The layer thickness $\delta$ for (a) $10 \times 10$ grid and (b) $20 \times 20$ grid, calculated on $\phi=0.9$ contour $\left(\diamond\right.$ : UDS, ○: SUDS). The solid line denotes the fitting function $\delta=c\left(s_{0}+s\right)^{1 / 4}$ where $c=0.14$, $s_{0}=-0.06$ for (a) and $c=0.076, s_{0}=-0.06$ for (b).

$c=0.14, s_{0}=-0.06$ for $10 \times 10$ grid, Figure $4(\mathrm{a})$, and $c=0.07, s_{0}=-0.06$ for $20 \times 20$ grid, Figure 4(b), respectively. The effects of lateral diffusion would be reduced if high-order central difference approximation was used [7].

4.1.2. Unsteady case. In addition to the quantitative comparisons for steady solutions, we also demonstrate the behaviour of the SUDS in a transient test case. The numerical setup is the same as that in the previous steady case but we want to look at the initial transient. In the exact solution, the step at west boundary moves along the $45^{\circ}$ diagonal. The Euler explicit scheme (perhaps not the best choice) was used. Figures 5 and 6 show the contours of $\phi$ calculated on a $20 \times 20$ grid for UDS, CDS and SUDS at time $t=0.2 L / U_{0}$ and $t=0.4 L / U_{0}$, respectively. CDS gives us oscillatory results which have comparable magnitudes (in the order of $O\left(10^{-1}\right)$ ). Very mild oscillation is observed (in the order of $O\left(10^{-3}\right)$ ) in the SUDS case while the UDS provides smooth and monotonic solution. No significant lateral spreading is observed in SUDS, consistent with our discussion in the last section. The UDS results generate excess diffusion initially $\left(t=0.2 L / U_{0}\right.$, Figure 5(a)) and later the transient contours $\left(t=0.4 L / U_{0}\right.$, Figure 6(a)) resemble the steady solution in Figure 3(a). It is evident that UDS produce excess diffusion in the transient flows. At the outflow boundaries, we can observe the effects of first-order upwind scheme in all cases. In general, the transient behaviour of the SUDS is much closer to that of the based scheme (second-order central difference in our case) we choose. The role of anisotropic diffusion is mainly to stabilize the numerical procedure at each time step instead of producing monotonic solution using standard upwind-type methods. Standard upwinding of the convective term is usually not consistent with centrally weighted source and transient terms, resulting in excessively diffusive solutions [3,8]. In SUDS, a great amount of anisotropic diffusion also implies that increased resolution may be required in some regions. 


\section{J. H. FERZIGER AND Y.-H. TSENG}

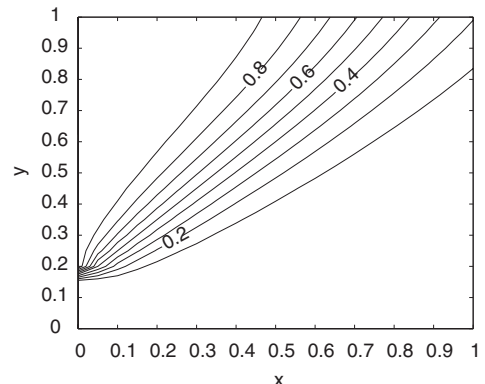

(a)

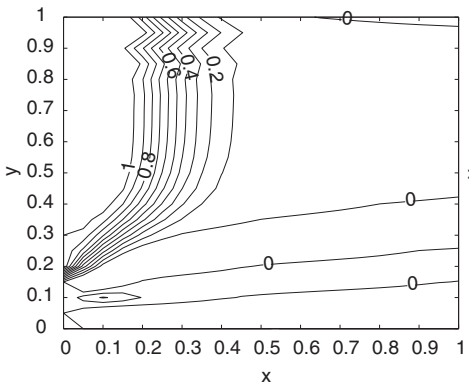

(b)

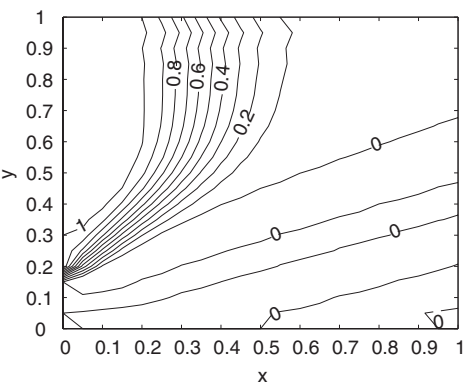

(c)

Figure 5. Contour plot of the scalar $\phi$ for a constant convective flow on a $20 \times 20$ grid at $t=0.2 L / U_{0}$ : (a) UDS; (b) CDS; and (c) SUDS.

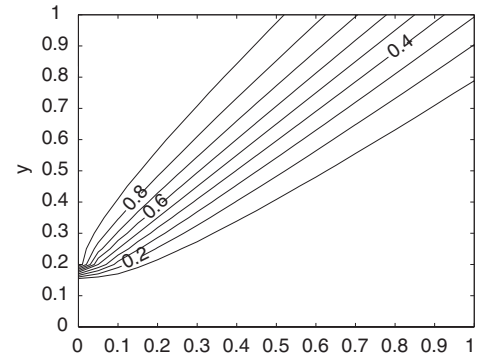

(a)

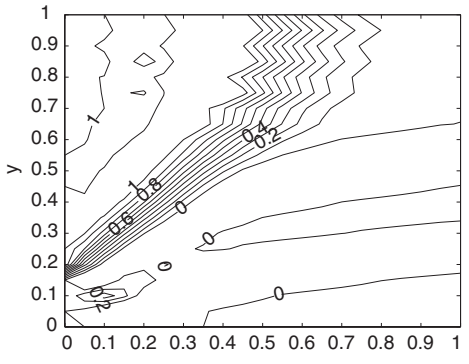

(b)

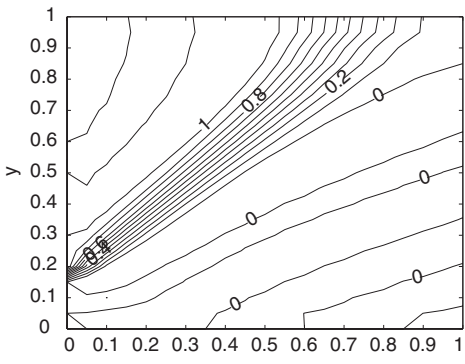

(c)

Figure 6. Contour plot of the scalar $\phi$ for a constant convective flow on a $20 \times 20$ grid at $t=0.4 L / U_{0}$ : (a) UDS; (b) CDS; and (c) SUDS.

\subsection{Scalar transport in a stagnation flow}

The second example involves the transport of scalar quantity in a more complex velocity field, one with curved streamlines. The boundary condition for scalar $\phi$ is still the step profile on the inflow boundary $x=0$ but the step is located at $y=0.5$. The velocity field is given by $u=1-x$ and $v=y$, which represents the flow near a stagnation point (the exact solution is provided in Figure $7(\mathrm{a}))$. The streamlines are the lines $(1-x) y=\mathrm{const}$, and change direction with respect to the Cartesian grid. The contours of $\phi$ calculated on $20 \times 20$ and $40 \times 40$ uniform grids for UDS, CDS and SUDS are presented in Figures 7 and 8, respectively. The contour plots clearly show the effects of numerical diffusion and dispersion. In CDS, strong oscillations over the whole domain result from the step change in $\phi$ along the inflow boundary.

It is clear that the oscillation in CDS propagates mainly in the streamwise direction. Adding sufficient diffusion in this direction smoothes the results significantly. The spread of contour lines shows the effect of numerical diffusion introduced by the schemes. It is obvious that the numerical diffusion from UDS is much greater than that from SUDS. No numerical diffusion is observed in CDS but the severe dispersion error results in large oscillations in a significant 


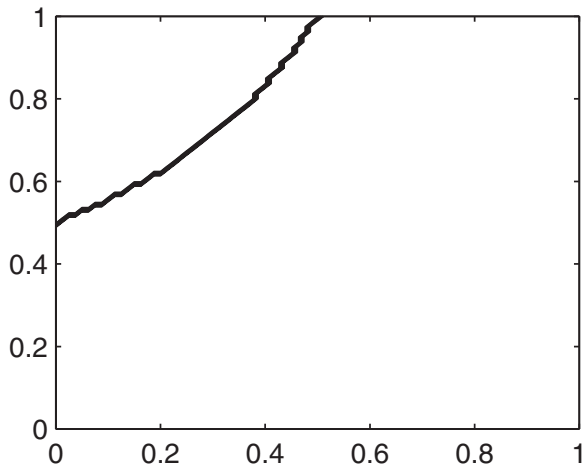

(a)

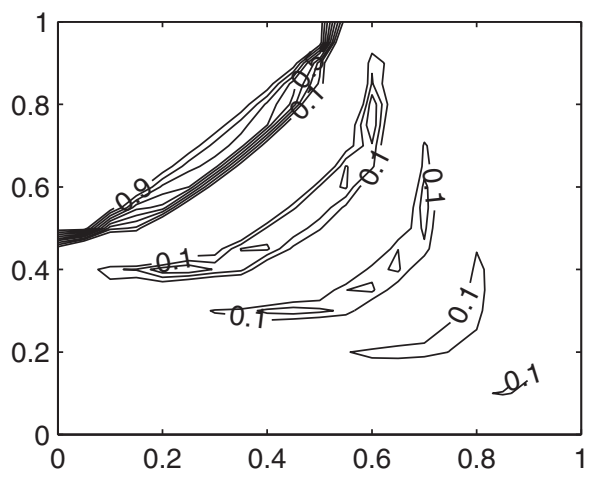

(c)

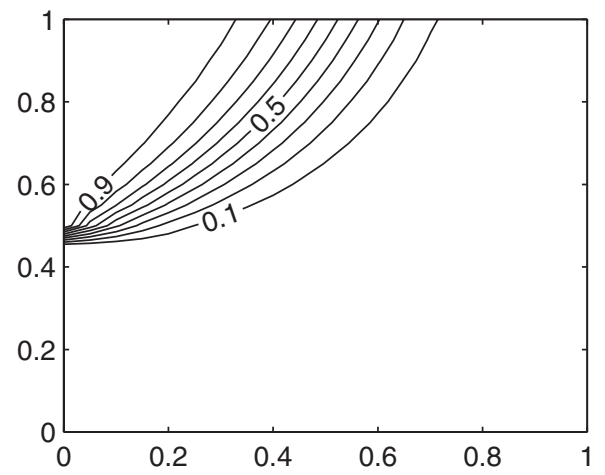

(b)

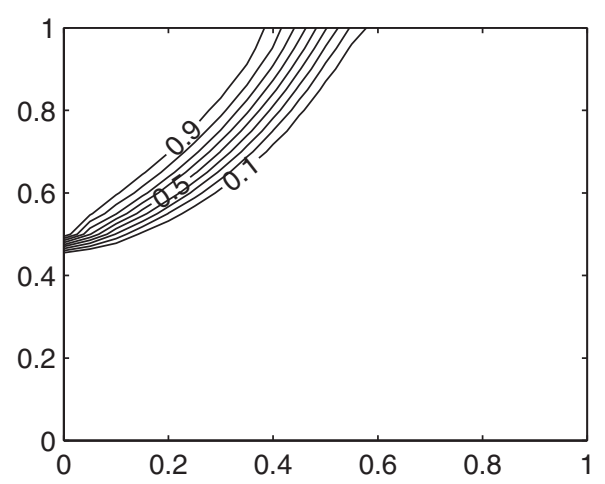

(d)

Figure 7. Contour of $\phi$ near a stagnation point with a step profile at the west boundary on a $20 \times 20$ grid. Comparison among different schemes: (a) Exact solution; (b) UDS solution; (c) CDS solution; and (d) SUDS solution.

part of the domain. Both CDS and SUDS results converge toward the exact solution faster than UDS. When the computational grid is fine enough, the local Peclet number is reduced and the introduced diffusion in SUDS converges to zero. Thus SUDS results will converge to the exact solution.

We also show the profile of $\phi$ at $x=0.75$ on uniform $10 \times 10$ and $20 \times 20$ grids in Figure 9 . The CDS solution contains very strong oscillations while, as expected, the UDS solution does not oscillate. However, the latter is much too diffuse. SUDS produces a profile with much improved steepness and generates only a very small oscillation. The major issue for SUDS is the balance between accuracy and stability. The criteria used to generate the diffusivity for SUDS does not guarantee monotonicity; it merely eliminates $2 \Delta x$ waves.

\subsection{Lid-driven cavity flow}

Finally, we selected a classic example to illustrate the streamwise upwind method for incompressible flow. The numerical method described in last section is applied to a laminar lid-driven cavity flow at Reynolds number $\operatorname{Re}=\rho U_{\mathrm{L}} H / v=2000$, where lid velocity is $U_{\mathrm{L}}$ 


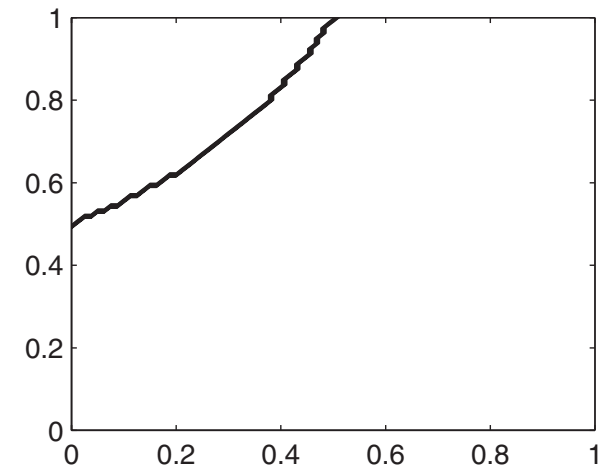

(a)

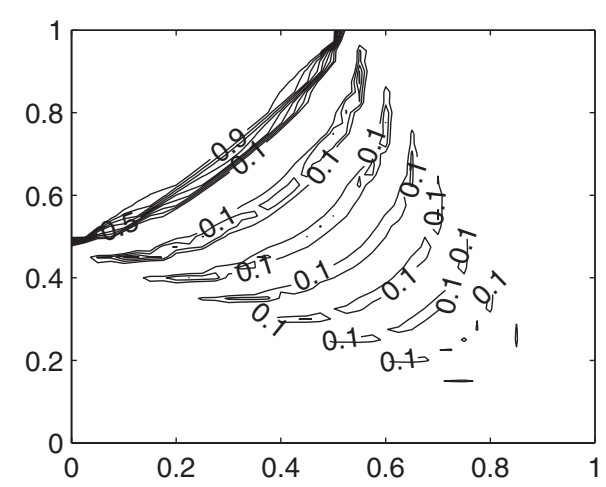

(c)

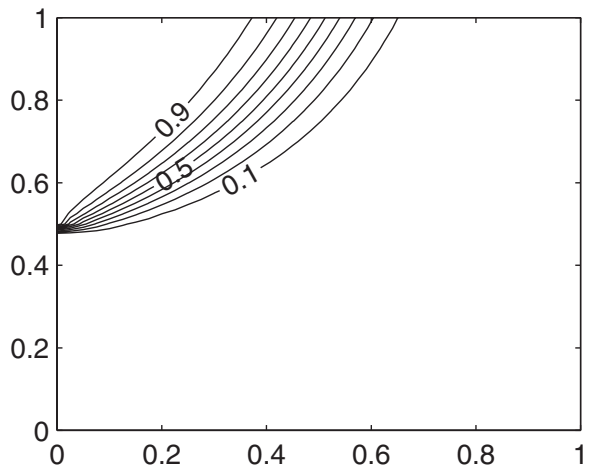

(b)

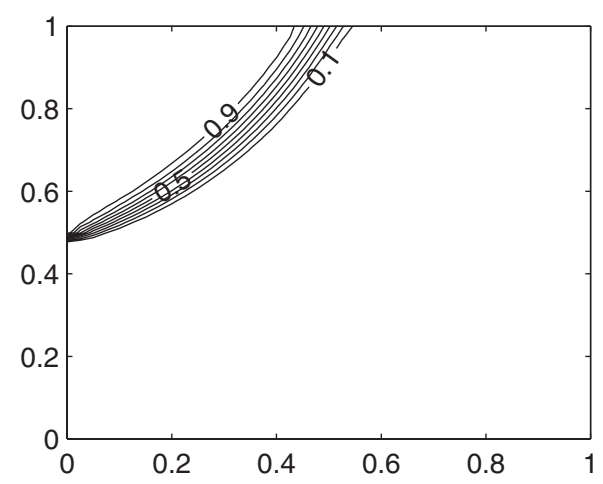

(d)

Figure 8. Contour of $\phi$ near a stagnation point with a step profile at the west boundary on a $40 \times 40$ grid. Comparison among different schemes: (a) Exact solution; (b) UDS solution; (c) CDS solution; and (d) SUDS solution.

and cavity height is $H$. This case has been used by many authors and accurate solutions are available in the literature. More discussion can be found in Reference [9]. Comparisons of the profiles of these horizontal (vertical) velocities at the vertical (horizontal) centreline are shown in Figures 10 and 11, respectively. The grid resolution varies from $10 \times 10$ to $160 \times 160$. UDS is not very accurate and the solution on the finest grid is still far from the grid independent solution. Both CDS and SUDS show monotonic convergence and the results are very similar. They both show second-order accuracy in the convergence error (Figure 12) while UDS shows only first-order accuracy. This is expected since the numerical diffusion from SUDS is reduced in a second-order manner as the grid is refined.

One of the major benefits of SUDS is that it removes the oscillation from CDS along the streamwise direction on the coarse grid. This can be seen by plotting the vertical velocity on a vertical line at $x=0.1$ very close to the west wall (Figure 13). CDS generates a moderate oscillation near the upper lid on the coarse grid (grid resolution: $20 \times 20$ ) while SUDS removes the oscillation by adding a small amount of diffusion but maintains the accuracy of CDS. 
CO-ORDINATE SYSTEM INDEPENDENT STREAMWISE UPWIND METHOD

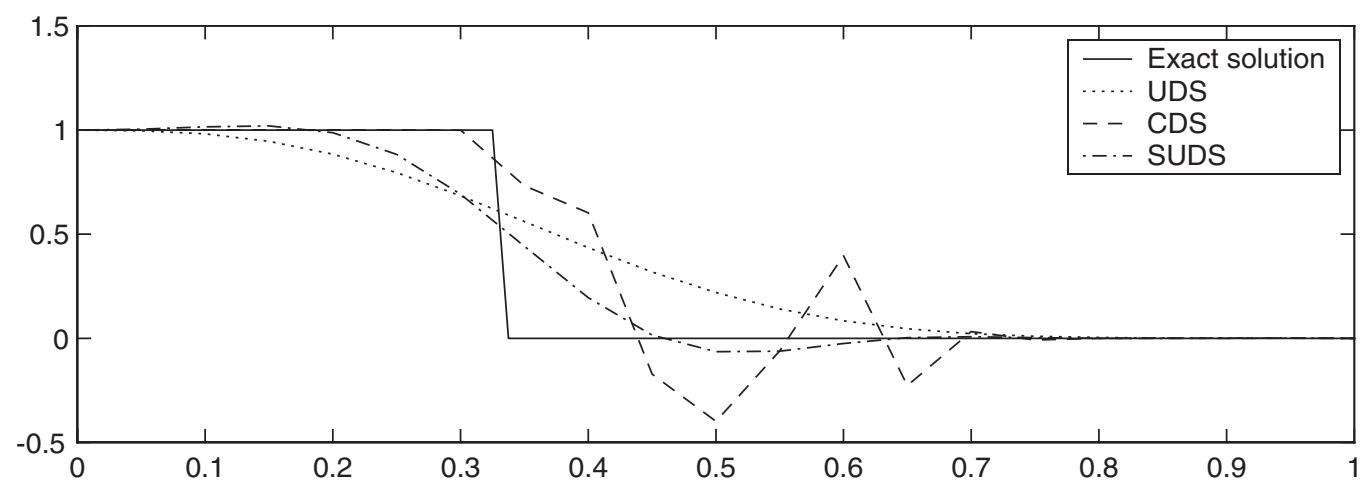

(a)

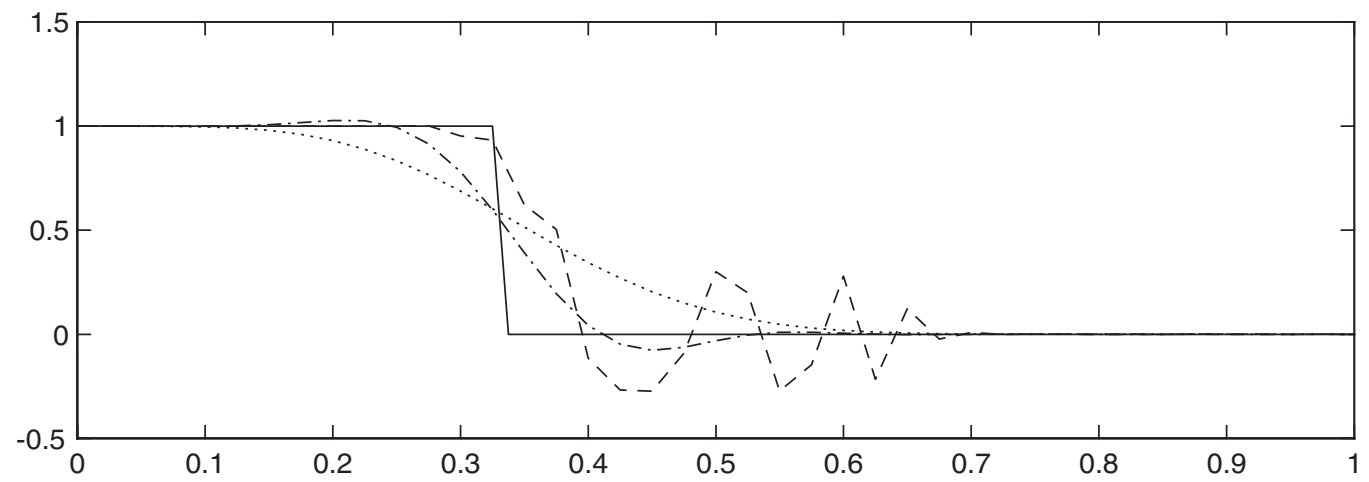

(b)

Figure 9. Profile of $\phi$ at $x=0.75$, calculated on a $10 \times 10$ grid (a) and a $20 \times 20$ grid (b).

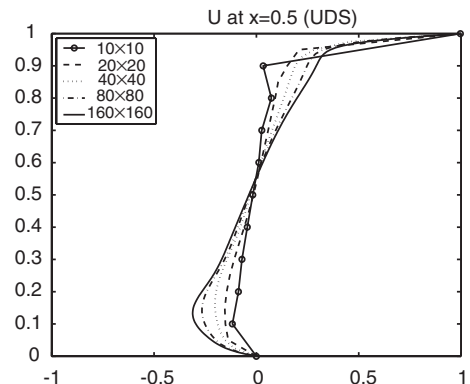

(a)

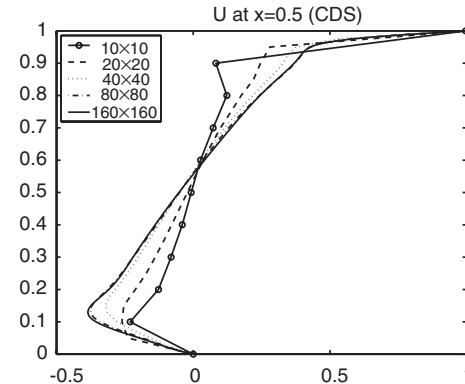

(b)

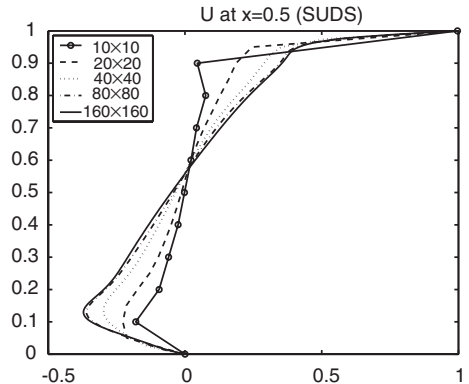

(c)

Figure 10. Velocity profiles at the vertical centreline at $R e=2000$, calculated on various grids ranging from $10 \times 10$ to $160 \times 160$ : (a) UDS; (b) CDS; and (c) SUDS. 


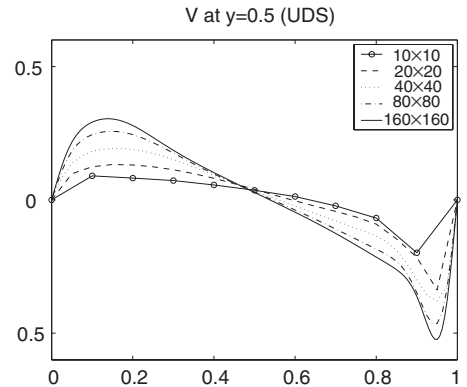

(a)

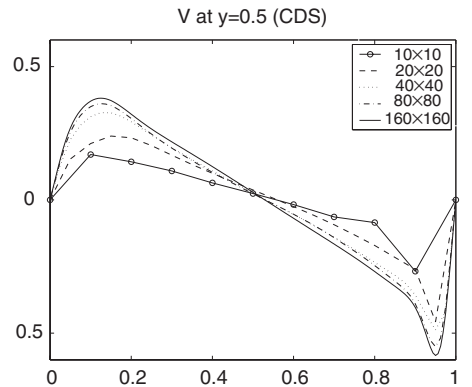

(b)

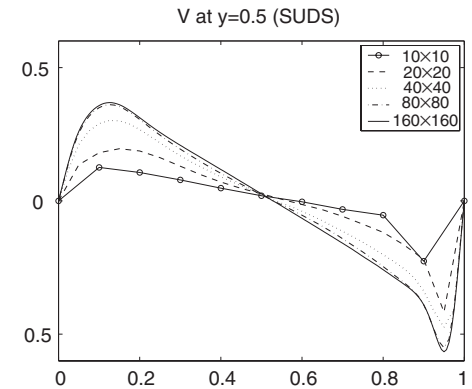

(c)

Figure 11. Velocity profiles at the horizontal centreline at $R e=2000$, calculated on various grids ranging from $10 \times 10$ to $160 \times 160$ : (a) UDS; (b) CDS; and (c) SUDS.

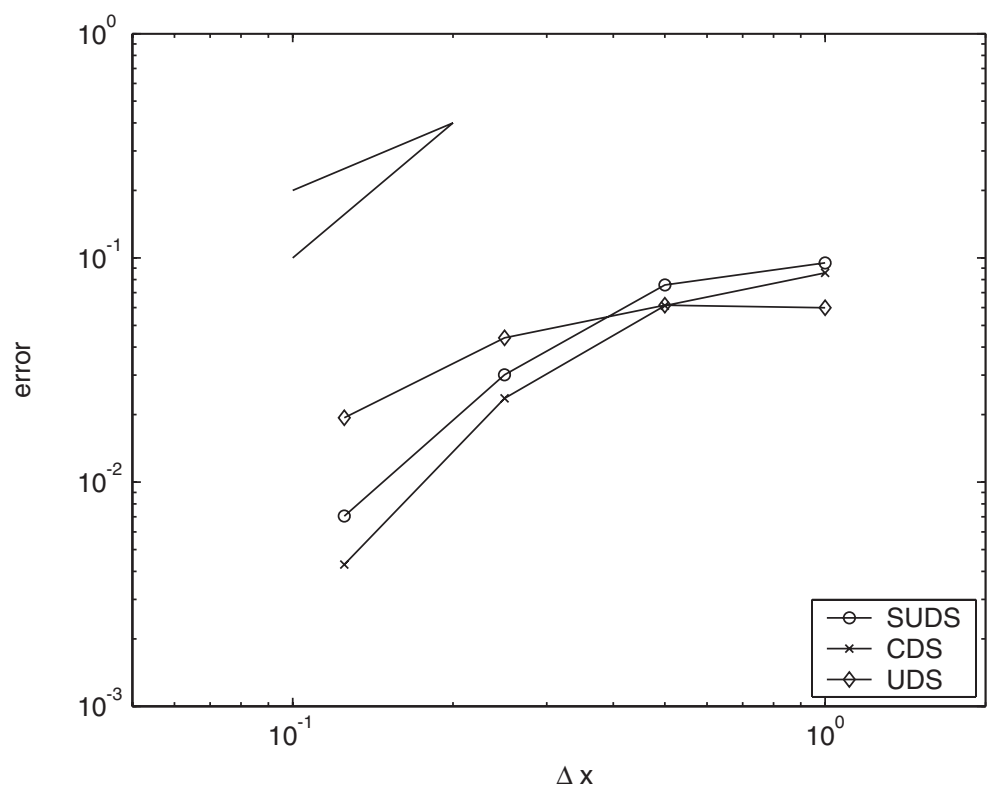

Figure 12. Comparison of the convergence error for the velocity in a lid-driven cavity flow at $R e=2000$ for different convective schemes. The upper notation $>$ represents slope -1 and -2 .

\section{SUMMARY AND CONCLUSIONS}

This study has purposed a new co-ordinate invariant streamwise upwind formulation. The damping of high-frequency dispersion error (oscillations) is achieved by adding a co-ordinate invariant anisotropic eddy diffusivity/viscosity tensor to the equations in accord with the local cell Peclet number. The method adds anisotropic diffusivity/viscosity only in the streamwise direction and possesses the desirable features of accuracy and stability. It also eliminates 


\section{CO-ORDINATE SYSTEM INDEPENDENT STREAMWISE UPWIND METHOD}

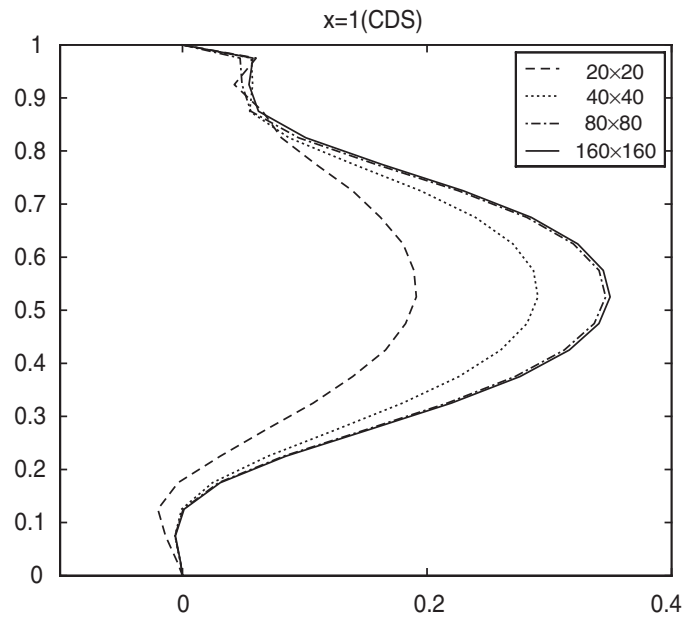

(a)

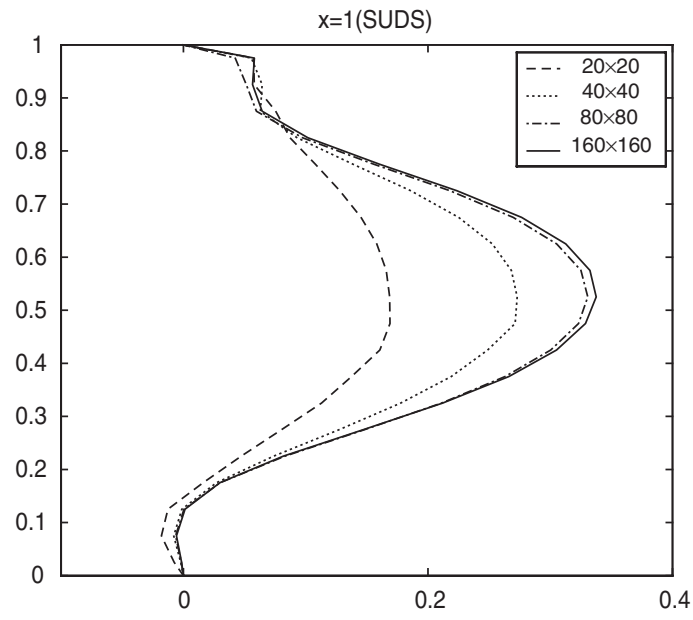

(b)

Figure 13. Velocity profiles at the horizontal centreline at $R e=2000$, calculated on various grids ranging from $10 \times 10$ to $160 \times 160$ : (a) UDS; (b) CDS; and (c) SUDS.

the excessive crosswind diffusion of other upwind-biased dissipative schemes. We used a constant convective flow to demonstrate the $\frac{1}{4}$ power lateral spreading of the current scheme. A stagnation flow and a lid-driven cavity flow are used to illustrate the accuracy and the properties of the method. The method is easy to implement and retains the accuracy of the chosen numerical scheme. The extension to three-dimensions is straightforward.

\section{REFERENCES}

1. Kreiss HO, Oliger J. Stability of the Fourier method. SIAM Journal on Numerical Analysis 1979; 16:421-433.

2. Dietrich DE, Mehra A, Haney RL, Bowman MJ, Tseng YH. Dissipation effects in North Atlantic Ocean modeling. Geophysical Research Letters 31:L05302. DOI: 10.1024/2003GL019015.

3. Brooks AN, Hughes TJR. Streamline upwind Petrov-Galerkin formulations for convection dominated flows with particular emphasis on the incompressible Navier-Stokes equations. Computer Methods in Applied Mechanics and Engineering 1982; 32:199-259.

4. Kelly DW, Nakazawa S, Zienkiewicz OC, Heinrich JC. A note on upwinding and anisotropic balancing dissipation in finite element approximations to convective diffusion problems. International Journal for Numerical Methods in Engineering 1980; 15:1705-1711.

5. Raithby GD. Skew upstream differencing schemes for problems involving fluid flow. Computer Methods in Applied Mechanics and Engineering 1976; 9:153-164.

6. Large WG, Danabasoglu G, McWilliams JC, Gent PR, Bryan FO. Equatorial circulation of a global ocean climate model with anisotropic horizontal viscosity. Journal of Physical Oceanography 2001; 31:518-536.

7. Morinishi Y, Lund TS, Vasilyev OV, Moin P. Fully conservative higher order difference schemes for incompressible flow. Journal of Computational Physics 1998; 143:90-124.

8. Gresho PM, Lee RL. Don't suppress the wiggles - they're telling you something. Computers and Fluids 1981; 9:223-253.

9. Ferziger JH, Perić M. Computational Methods for Fluid Dynamics (3rd edn). Springer: Berlin, Heidelberg; 2001 . 